\title{
Active Reflectors: Possible Solutions Based on Reflectarrays and Fresnel Reflectors
}

\author{
Lorena Cabria, ${ }^{1}$ José Ángel García, ${ }^{1}$ Julio Gutiérrez-Ríos, ${ }^{2}$ \\ Antonio Tazón, ${ }^{1}$ and Juan Vassal'lo ${ }^{3}$ \\ ${ }^{1}$ Departamento de Ingeniería de Comunicaciones, Universidad de Cantabria, Avenida los Castros s/n, 39005 Santander, Spain \\ ${ }^{2}$ Departamento de Tecnología Fotónica, Universidad Politécnica de Madrid, Campus Montegancedo, \\ 28660 Boadilla del Monte Madrid, Spain \\ ${ }^{3}$ Instituto de Física Aplicada, Consejo Superior de Investigaciones Científicas, Serrano 144, 28006 Madrid, Spain \\ Correspondence should be addressed to Juan Vassal'lo, ltqvs22@ifa.cetef.csic.es
}

Received 24 September 2008; Revised 28 November 2008; Accepted 4 February 2009

Recommended by Giovanni Toso

\begin{abstract}
An overview about some of the recent Spanish developments on active reflectors is presented. In the first part, a novel beamsteering active reflectarray is deeply studied. It is based on implementing in each elementary radiator an IQ modulator structure, in which amplitude and phase control of the scattered field is achieved. Finally, a special effort is made in offering solutions to overcome the active antenna integration problems. In the second part, the active concept is firstly extended to Fresnel reflectors. Thanks to the development of a proper simulator, this special structure can be easily analysed. This simulator allows the study of performance of this kind of reflectors and, applying evolutionary algorithms, to find optimal configurations of reflector in accordance with the given specifications for the conformal radiation pattern.
\end{abstract}

Copyright (C) 2009 Lorena Cabria et al. This is an open access article distributed under the Creative Commons Attribution License, which permits unrestricted use, distribution, and reproduction in any medium, provided the original work is properly cited.

\section{Introduction}

Nowadays, the development of antennas with reconfigurable beam pattern capabilities represents one of the keys issues for the future of the telecommunications and remote sensing applications. Considering this aspect, this paper proposes two different reconfigurable reflectors: an active reflectarray and a Fresnel reflector. The reflectarray is formed by resonant microstrip patches coupled to an active circuit which provides the amplitude and phase control of the reflected signal. The Fresnel reflector is based on the use of small reflecting cells, integrating each of them a mechanical control to regulate its position with respect to a reference one.

A reflectarray is a type of antenna that combines the best features of conventional reflector with those of the phased printed arrays. In this structure a horn or small antenna array illuminates a planar array of microstrip radiators, which, by using suitable phasing circuits, converts an incident spherical wave into a plane wave, collimating the reflected field in a desired direction [1]. Their low cost, flatness, and easiness to install and manufacture convert these structures into ideal solutions to modern communication systems [2]. Moreover, spatial signal distribution avoids the complexity and losses associated to the microstrip feeding distribution network.

The possibility of employing active antennas as reflectarray cells offers additional advantages derived from their power combining features. In this sense, amplifying functions have been already added in individually phased active antenna elements [3]. Nevertheless, only a few works have considered the possibility of varying the scattered field phase from each element, achieving beam steering capabilities [4].

The control over the capacitance, obtained in [4] through the use of a varactor diode, has been also proposed for the design of reconfigurable reflectarrays with scanning capability, either serially connecting two halves of a microstrip patch [5] or phase tuning a high-impedance surface [6]. MEMS are also standing as a promising solution for the design of tuneable reflectarrays, mainly in terms of linearity, power consumption, losses, and size, as suggested from recent results [7].

Despite all their advantages, some problems have to be faced when designing or implementing active reflectarrays. 
As a way of illustration, if high gain amplifying stages were employed between the orthogonal feeds of a patch, a poor out of band isolation between feeds might lead to amplifier oscillations. In this type of solutions, attention should be put to the radiator isolation characteristic versus frequency as well as to the detection of instabilities through the use of linear [8] but also nonlinear analysis techniques. In transmitting reflectarrays, especially when power amplifiers are integrated, especial attention should be put to thermal aspects. The available heat sink capability for power dissipation would impose a limit to the device output power level. The introduction of novel highly efficient technologies, as the case of GaN HEMTs, or the application of emerging transmitting concepts, could help overcoming this power handling limit.

With respect to Fresnel reflectors, as it is well-known, their surface efficiency cannot be greater than the provided by a parabolic reflector [9-11]. Nevertheless, they can offer advantages that a parabola cannot provide, as for example, the feeder of a Fresnel reflector can a change of position, out the focal point, without any important variation on performance.

This property is used to develop an active Fresnel reflector, placing a distribution of reflecting cells over a flat surface. Each one of these cells is coupled to an active mechanism which allows a change of position with respect to the feeder. So, any distribution of Fresnel zones can be configured over the whole reflecting surface.

The inclusion of active elements on these kinds of antennas (reflectarrays and Fresnel reflectors) provides a control over the antenna radiation pattern in the reflecting surface. So, the designer can work with a new freedom degree if this possibility is added to the classical control on the feeder.

\section{Active Reflectarray Development}

In this paper, an active reflectarray with attractive capability of varying the pencil-beam or shaped beam radiation pattern is presented. Based on the vector sum method, the amplitude and phase of the reradiated field from each elementary radiator is controlled by means of applying a DC voltage through the IF port of two double balanced diode mixers in an IQ configuration. This kind of structure can provide a new freedom degree, because the steering can be found from a control of active circuits in the feeder (as in a not very old manner) and also be included in the reflector surface.

By means of an accurate adjustment of each element phase shift, it is possible not only to convert the spherical wave into plane one but also to steer the main beam. In this sense, in order to demonstrate the proposed radiating architecture potentialities, different radiation patterns have been measured.

As the idea is to put in evidence advantages and drawbacks of this kind of antenna, a laboratory demonstrator was designed, manufactured, and measured. To optimize time and efforts, the selected reflectarray was designed to have a planar configuration of $4 \times 2$ radiating elements.

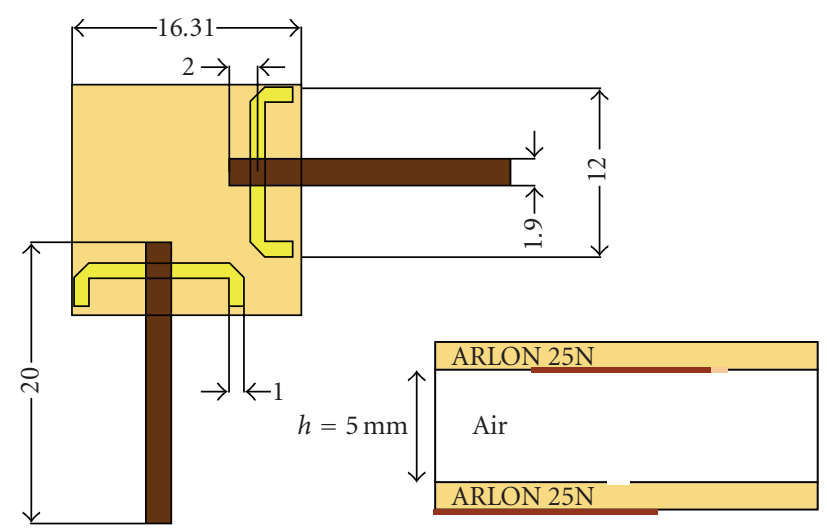

Figure 1: Top and side view of the designed square microstrip patch.

2.1. Radiating Element. An aperture coupled microstrip patch [12] with two orthogonal feeds has been selected as a reflectarray elementary radiator. One of its ports is employed to receive the signal from the feeder while the other is used to retransmit it with the amplitude and phase selected in the vector summing unit. Therefore, the square patch has two microstrip feeding inputs placed at perpendicular sides, exciting the TM01 and TM10 orthogonal modes for the same resonating frequency, aimed to the simultaneous reception and retransmission of the microwave signals using orthogonal polarizations. The dual feeding provides a reasonable isolation between its two ports, which is an important characteristic to be achieved when active elements are incorporated. The gain of the active path, from reception to retransmission, is limited by the isolation between both input ports, achieving an unstable state when such condition is not fulfillled.

Figure 1 illustrates the designed square microstrip patch. The active circuit and the feed microstrip lines have been printed in substrate ARLON $25 \mathrm{~N}$ with $\varepsilon_{r}=3.38$ and thickness of 30 miles $(0.762 \mathrm{~mm})$. In order to improve the gain and the bandwidth, the air is employed as radiator substrate. Consequently, an auxiliary layer is needed to print the patch on it. The working frequency band, around $5.8 \mathrm{GHz}$ in our particular application, determines the dimensions.

A commercial electromagnetic simulator (Ansoft Ensemble) has been employed to design and analyze the structure. In Figure 2, the simulated and measured input matching and isolation between both ports are shown.

2.2. Amplitude and Phase Control Unit. Figure 3 shows a scheme of the amplitude/phase control unit implemented in each elementary radiator. The received signal is equally divided through a $90^{\circ}$ hybrid coupler exciting the LO ports of a pair of double balanced diode mixers. By proper adjustment of the DC voltage, applied in the IF port, it is possible to vary the amplitude and to switch the phase, between two values with a $180^{\circ}$ shift (depending on the DC sign) of the RF signals. Finally, these output signals are combined employing a Wilkinson circuit achieving a complete $\left(360^{\circ}\right)$ phase control range. 


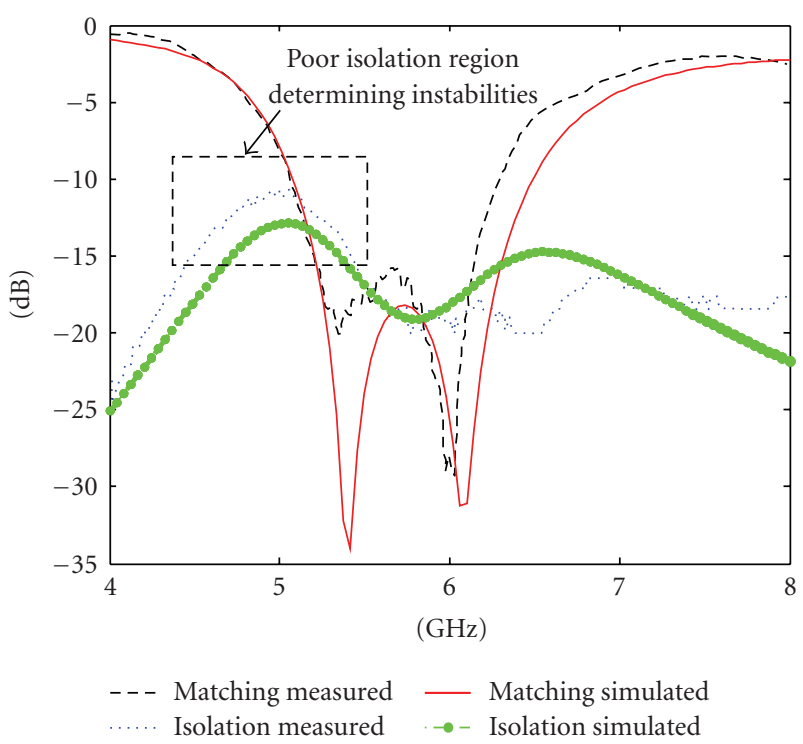

FIGURE 2: Measured and simulated input matching and isolation.

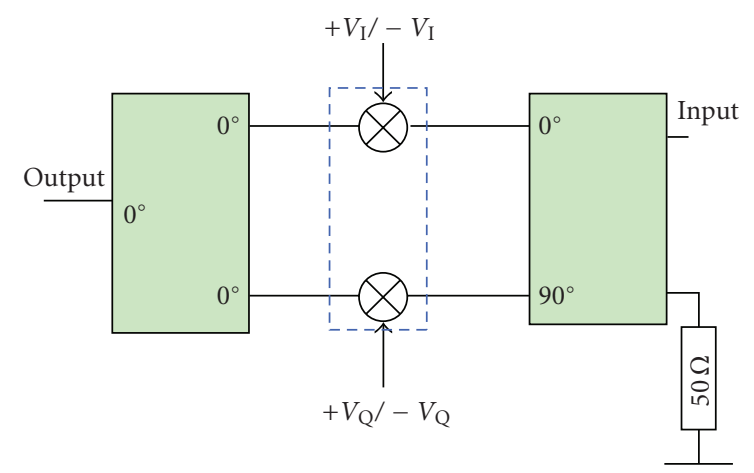

Figure 3: Amplitude/phase control unit used in each reflectarray elementary radiator.

In order to clarify this behavior, a summing scheme can be used. The output signal is the resultant vector obtained through a sum of two perpendicular variable vectors. Consequently, using a pair of conveniently selected voltage values, the magnitude and phase of the resultant vector may be controlled in a complete phase range.

\subsection{Integration of the Active Circuits on the Radiating Element.} The radiating element and the amplitude/phase control circuitry were integrated in the same $\mathrm{PCB}$ employing the (Active Integrated Antenna (AIA)) concept to minimize the cable and feeding line losses in a similar way to [13].

In order to compensate the associated attenuation of the IQ control circuit two commercial amplifiers were added in each unit. In Figure 4, a clarifying schematic as well as a detailed photograph of the active elementary radiating unit can be seen.

In the photograph some, parts of the reflectarray unit have been highlighted. The MBA-671 mixers from Minicircuits, and the NBB 400 amplifiers from RF Microdevices constitute the active parts with a power consumption of $665 \mathrm{~mW}$. In the IQ structure, the input $90^{\circ}$ hybrid coupler is a high-quality and small size commercial circuit from Anaren, while the combination of the output signals of each mixer was done through a microstrip Wilkinson circuit specifically designed for it. To accurately adjust the phase/gain of each circuit, different SMA connectors were added to take samples of the signal in different parts.

The active control circuit was measured independently of the patch obtaining a maximum gain of $12 \mathrm{~dB}$. Complete phase range was also measured for different gains.

Once the structure was measured with the SMA connectors and its correct performance verified, the complete radiating structure was probed appearing undesired oscillating frequencies around $5 \mathrm{GHz}$. The problem came associated to the poor isolation between the antenna ports at this band of frequencies, comparable to the gain of the active path, as it has been highlighted in Figure 2. Consequently, it was necessary to eliminate this unpleasant effect. Taking into account the limited space available in the reflectarray unit, a stub was added to reduce the coupling in the previously mentioned band, as it can be observed in Figure 4 .

2.4. Active Reflectarray Implementation. Figure 5 shows the layout of the reflectarray. In such antenna, the selected feeder is an aperture-coupled microstrip patch equal to the reflectarray radiating elements, which transmits a signal in the working band with vertical polarization. The reflectarray active circuits, not included in this illustration in order to clarify the whole radiating structure, were inserted in the feeding layer.

In order to avoid feed blockage-an offset feeding configuration was used, placing the feeder at $200 \mathrm{~mm}$ down from the center and $55 \mathrm{~mm}$ out from the array.

A photograph of the reflectarray feed layer is shown in Figure 6. As it has been previously commented, each unit has two varying bias voltages to control the mixers and one fixed voltage to supply the amplifiers.

Each element demands a phase shift value as a function of its position with respect to the feeder to convert the spherical incident wave to a planar one. There are different ways to achieve the required phase shift per cell: in [14] equal size microstrip patches are employed with passive coupled delay lines of different lengths, while in [15] the phase of the scattered field is controlled by means of the patch size. Although these methods permit to assure the correct antenna performance in a higher bandwidth, the proposed system takes advantage of the total phase control in each element provided by the active circuit. Additionally, the possibility of controlling the phase shift between microstrip patches allows the change of the main beam direction.

In such case, the field phase in each unit is calibrated independently to fulfill, (1) $[15,16]$ :

$$
\phi_{i}=k_{0} \cdot\left(R_{i}-\tilde{r}_{i} \cdot \tilde{r}_{0}\right),
$$

where $R_{i}$ is the distance from the feeder phase center to the element, $\tilde{r}_{i}$ is the vector from the array center to the element, and $\widetilde{r}_{0}$ is the unit vector in the main beam direction. 


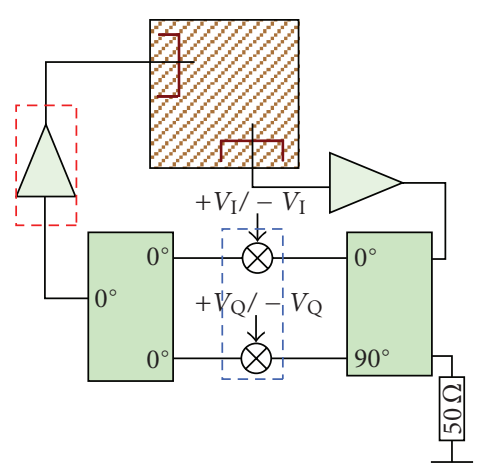

(a)

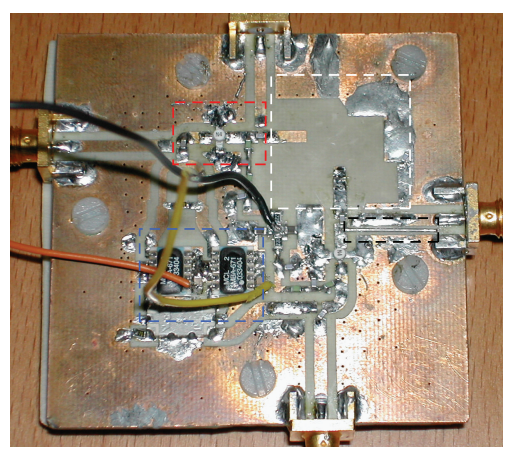

(b)

Figure 4: (a) Schematic and (b) photograph of the reflectarray unit.

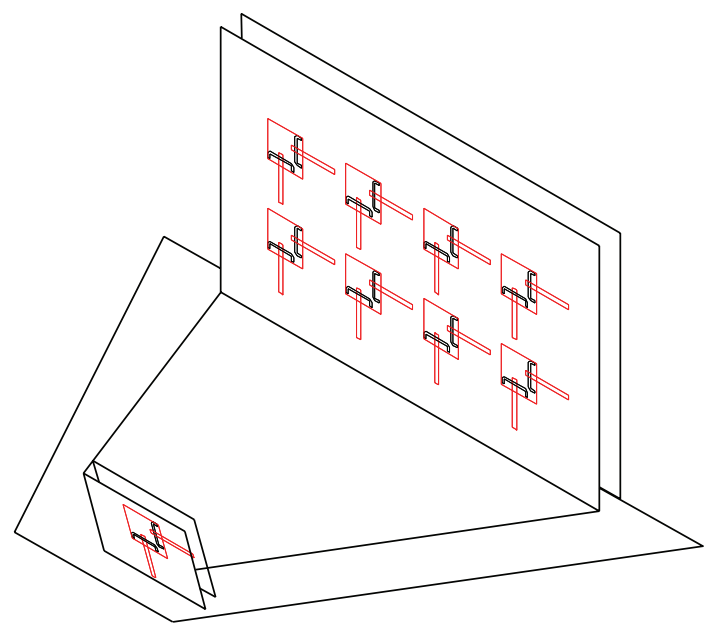

Figure 5: Layout of reflectarray, feeder included.

In order to vary the reflectarray beam pointing, the required phase shift values per radiating unit have to be recalculated following (1).

2.5. Measurement Results. A specific test setup was implemented in an anechoic chamber to measure the described antenna. In such scenario, a perfect calibrated horn antenna was employed in reception to measure the reflectarray response, assuring a far field distance between them. As it has been commented before, the radiation pattern phase evolution for each unit was calibrated in the main direction for different gains. In this way, it was possible to select those biasing points per unit to fulfill (1) for different pointing directions.

Due to the huge number of different controlling bias voltages and in order to generate a portable and compactness radiating structure, a special bias control circuitry was also implemented. The required voltages per unit, obtained in a previous step, were then introduced in a hexadecimal table in the $\mathrm{PC}$ as a function of the required gain and phase values. By means of an RS232 interface, the PC and the control board

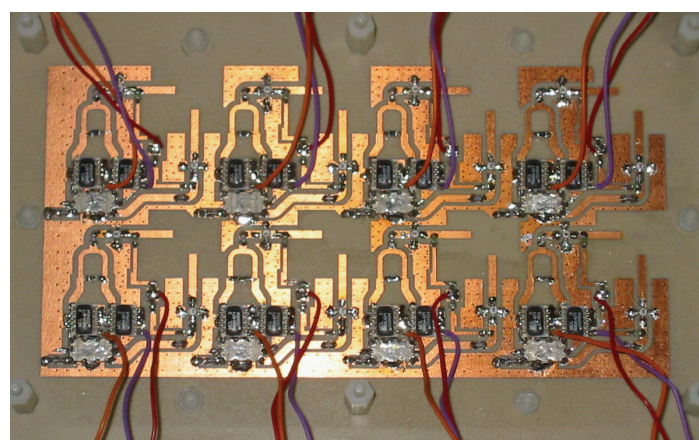

FIGURE 6: Reflectarray feeding layer.

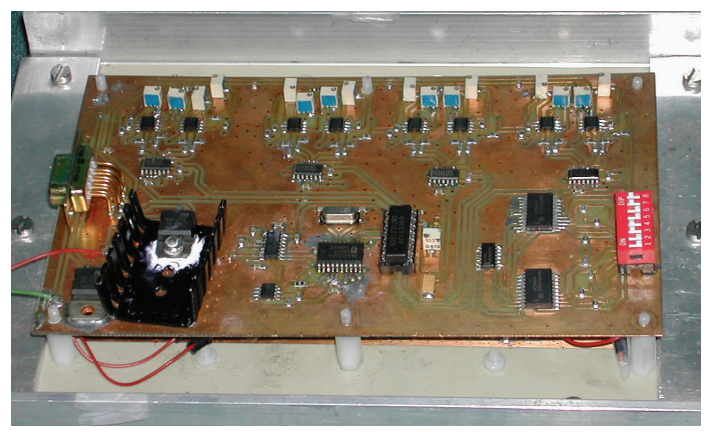

Figure 7: Rear view of the final manufactured prototype.

are connected, being able to provide the required bias to each mixer and amplifier. A rear view of the manufactured prototype can be seen in Figure 7.

Finally, the far field radiation pattern of the reflectarray was measured for three main pointing directions $\left(0^{\circ}, 10^{\circ}\right.$, $\left.-10^{\circ}\right)$. The individual amplitude control would also permit to conform different amplitude distributions if desired. In Figures 8, 9, and 10, measurements of normalized radiation patterns for a uniform amplitude distribution have been represented. A comparative study between the resultant measurements and the calculated array factor of the retransmitted patterns has been done and illustrated in these figures. 


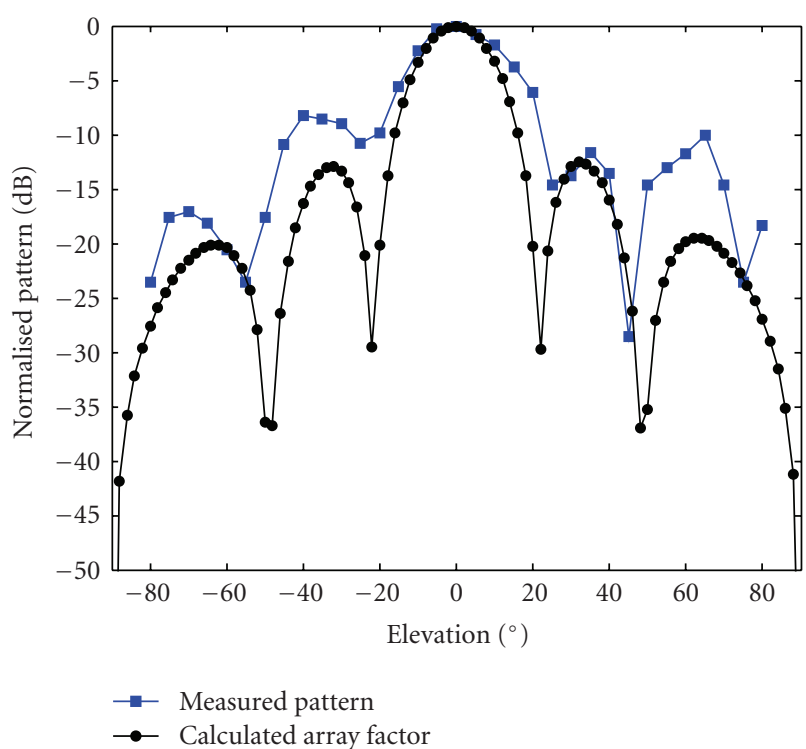

FIGURE 8: Measured and calculated reflectarray radiation pattern, pointing to $0^{\circ}$.

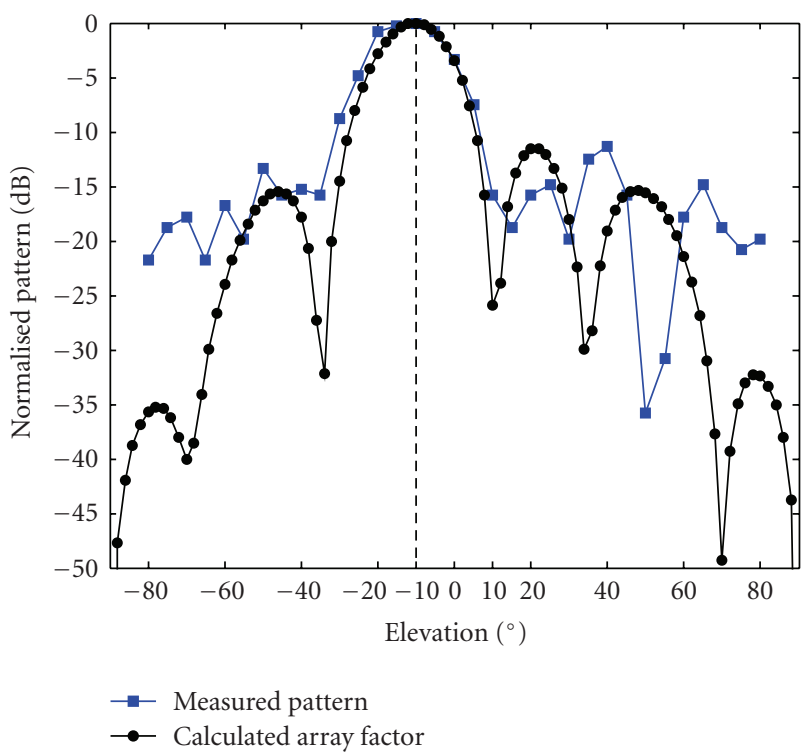

FIGURE 9: Measured and calculated reflectarray radiation pattern, pointing to $-10^{\circ}$.

As it can be seen, the similarity among the obtained results and the calculated ones demonstrates the potentiality of the proposed architecture.

2.6. Some Consequences of the Developed Prototype. As it can be seen in the photograph of the demonstrator, one of the drawbacks of the implementation of the active elements on the reflecting surface is the positioning of the control and feeding DC circuitry.

In a reflectarray of small number of elements (only eight radiators), the drawback can be easily solved as it can be seen in the photograph of the developed demonstrator. But

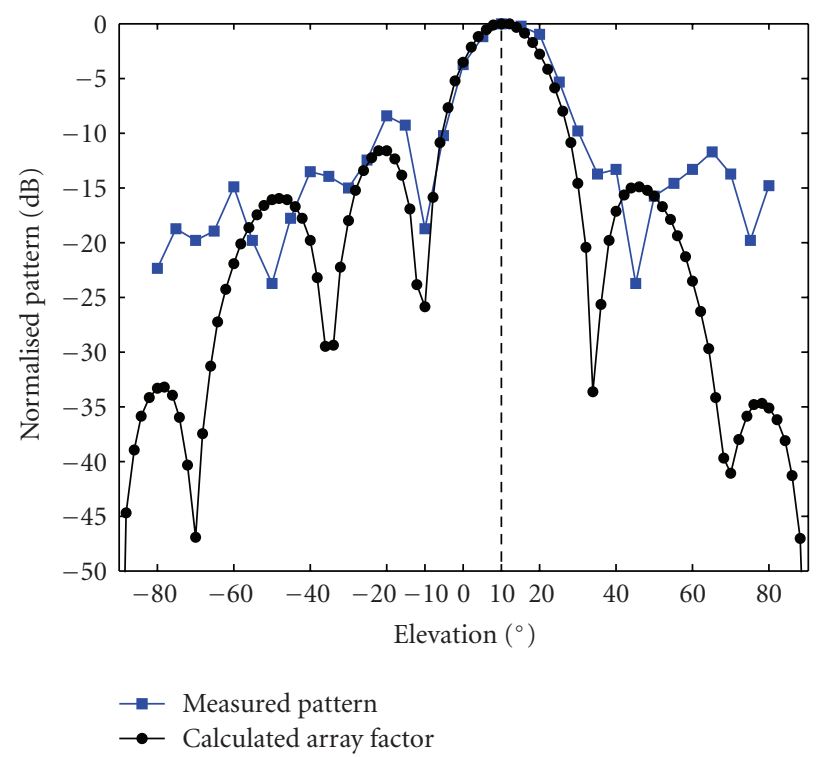

Figure 10: Measured and calculated reflectarray radiation pattern, pointing to $10^{\circ}$.

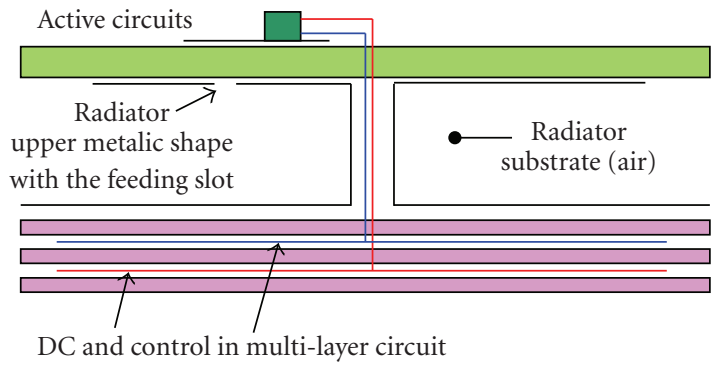

FIGURE 11: Structural concept to integrate DC and control circuit on active reflectarrays.

in the case of reflectarrays with high number of elements, it is necessary to achieve other kinds of solutions.

There are several possibilities now under development, for example, by means of a laser link between each module with a central unit or by using the DC circuitry as link of the control signal at low RF. Obviously, this solution has a better implementation by using a modular design.

Nevertheless, this restriction can be avoided if radiating elements are feeding from its radiating face. In this configuration, active circuits can be placed over the radiating face, and the control and DC circuitry can cross the microstrip patch through a metallic post placed on its center, because this central part of the radiator has a cero per impedance.

Then, the distribution circuitry of control and DC can be easily manufactured on a multilayer substrate and placed behind of the radiator microstrip ground plane. Figure 11 shows this solution.

\section{Active Fresnel Reflector Development}

Fresnel reflectors having arbitrary main beam transversal section, by means of conformed Fresnel zones, were 
presented in [17]. This possibility allows the design of a new concept on active reflectors, based on squared reflecting cells, each one coupled to a mechanical actuator. This configuration provides a degree of freedom about the position of each cell in the $z$-axis to be able to conform a good approximation to any desired Fresnel reflector geometry.

The idea that is explained in this paragraph is based on a flat structure formed by small reflectors, whose position is electronically controlled by means of mechanical actuators. So, a flat reflector with this configuration can work with any Fresnel distribution. The lattice of small reflecting cells is electronically controlled to provide any Fresnel distribution over the reflector surface and then to have a real control of the radiation pattern.

The number of mechanical actuators can be a problem of manufacturing, weight, and cost of the final prototype, and consequently it can constitute a limit for the approach of different radiation patterns to be generated. Nevertheless, in applications where the number of desired beams is small and previously known, the cell size can be optimized in order to reduce the number of actuators. The consequence is a final prototype of more easy manufacturing process and lower weight and cost.

Reflectors having Fresnel zones with axial symmetry have been analyzed in [18]. Results show that the behavior can be optimized depending of special parameter of this kind of reflectors, as for example, the number of levels of reflecting Fresnel zones. So, a specific simulator for Fresnel reflectors of squared cells has been developed, and its results are to be commented below.

\subsection{Active Fresnel Reflector}

3.1.1. Electrical Concept. The radiation pattern of a reflected signal can be modified by changes in the geometrical characteristics of the reflecting surface. As it is known, perturbations on parabolic surfaces allow any desired $3 \mathrm{~dB}$ transversal section in the main beam, being actually the best solution applied in geosatellite antennas for communication links.

This concept was translated in [17] to the Fresnel reflector technology, and a general behavior of Fresnel reflectors can be seen in [18], being the case of reflectors with 2 or 3 levels as the more interesting ones from performance and manufacturing point of view.

A Fresnel reflector design with 144 actuators of two positions, controlling an array of $12 \times 12$ reflecting cells, is to be described in this paper. The antenna has been designed to provide a beam scanning in the principal plane direction, having the main beam at the symmetric direction to the focal position of the feeder from the central point of reflecting surface (see Figure 12).

The selected working frequency was $18.75 \mathrm{GHz}$, which must be in accordance with the distance separation among levels, which is provided by the selected actuator. The cell size was selected taking into account the supposed application of the example, in order to avoid grating lobes and the area to be covered by the scanning. The desired beam scanning zone is between $0^{\circ}$ and $-20^{\circ}$, in the principal plane, which includes the focal point.

Once the geometry of the reference Fresnel configuration is determined, the movement of the synthesized geometry over the reflector surface will provide the beam scanning. So, the Fresnel configuration of reference must be synthesized over a surface bigger than that of the reflector. This allows the changes as it is shown in Figure 13, where there are three reflector configurations for different appointments.

It is obvious that in this structural concept, the feeder is always in the same position. The unique change on the antenna, which provides the desired scanning, is the change of the position of the Fresnel configuration on the reflector surface, as it can be seen in Figure 13. Figure 14 shows the superposition of simulated array factors of the Fresnel configurations to cover the selected scanning area. The evolution of the radiation pattern is shown in Figure 14, and consequently it can be understood that any reflected direction can be obtained by placing the Fresnel configuration at the adequate place over the reflector surface.

3.1.2. Mechanical Concept. To allow the change of Fresnel level of any reflecting cell, each Fresnel level is associated to a different position of the reflecting surface of the cell, thinking that this different position is referred to the perpendicular direction to the reflector surface.

To provide this position change, a commercial actuator (solenoids, series 45B) was selected from the web page of RS AMIDATA. Figure 15 shows the geometry data sheet.

As it can be seen in Figure 16, the actuator is placed under each reflecting cell, and it allows a displacement of the reflecting cell of $4.5 \mathrm{~mm}$, which is electronically controlled. This figure shows two complete active reflecting cells, at both states: 0 and 1, which corresponds to both Fresnel levels. The actuator is placed at the position 1 when there is a voltage of $6 \mathrm{~V}$ at the inputs of actuator terminal, and it is placed at the position 0 when the voltage is $0 \mathrm{~V}$. The actuator works over the reflecting cell as it can be seen in Figure 16.

When the aim is to implement reconfigurable Fresnel reflectors with more than two levels, the actuator should be different form that commercially available. However there is not a great technological problem, including a continuous shift solution. We can think about a little screw pin moved by a small electrical motor, step-by-step or continuous type.

Figures 17 and 18 show, a view of the whole Active Fresnel Reflector, respectively with the Fresnel configuration of Figures 13(b) and 12. The case that is shown in Figure 17 corresponds to the synthesized Fresnel configuration which is centered on the surface of the reflector.

3.2. Simulator of Fresnel Reflectors. Performance of Fresnel reflectors depends on the number of levels implemented but the result obtained with a three levels implementation is very reasonable. As a matter of fact, gain of a three levels reflector is usually about $1.5 \mathrm{~dB}$ under a same aperture and same focus paraboloid. However Fresnel reflectors have several important advantages: first, the fact being smaller in terms of occupied room. Second, the deployment system is 


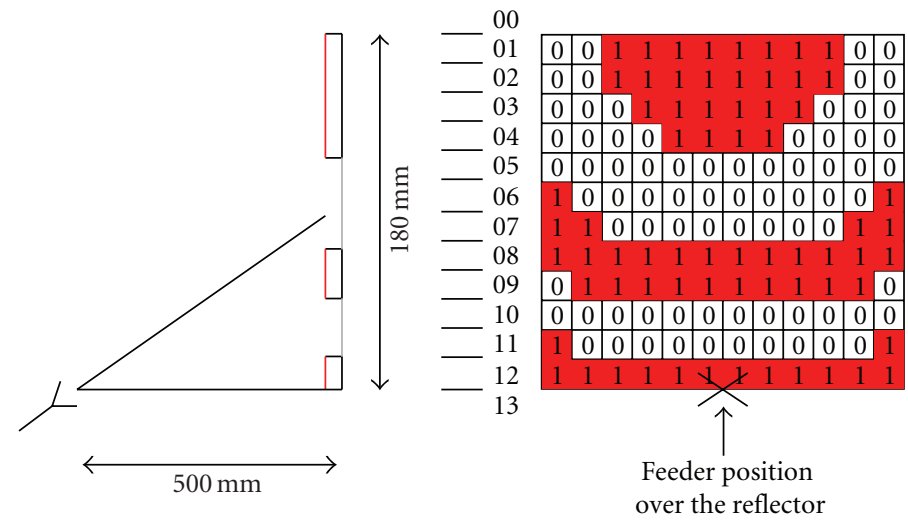

FigURE 12: Geometry of the synthesized Fresnel reflector configuration.

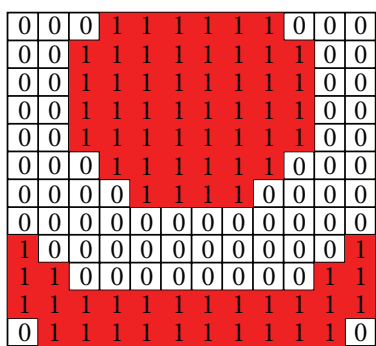

(a)

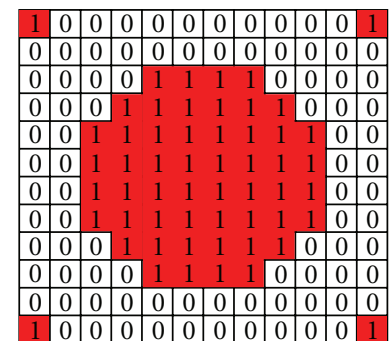

(b)

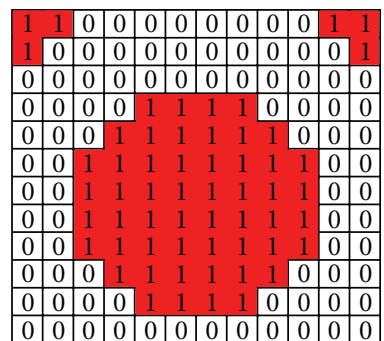

(c)

FIGURE 13: Reflector configuration for different positions of the Fresnel configuration.

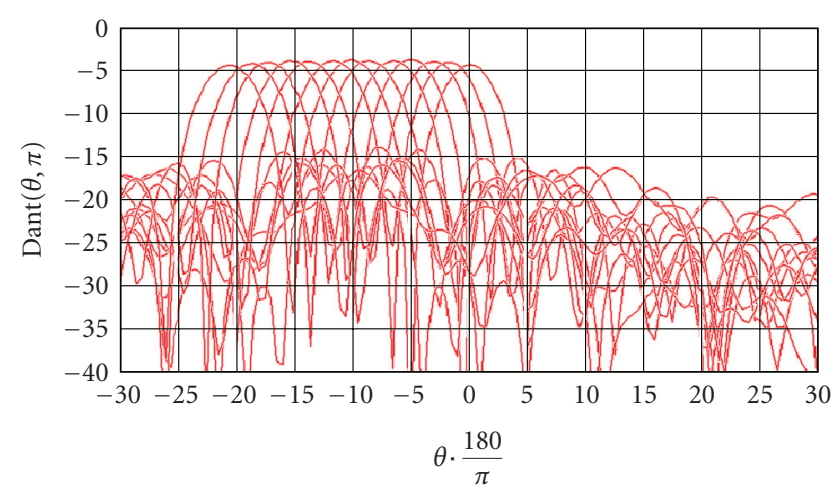

FIGURE 14: Superposition of simulated array factors, which are obtained by movement of the synthesized Fresnel distribution.

easier for them. Third, the possibility of combining several Fresnel distributions in a same reflector surface to get multibeam radiation patterns. Finally, the manufacturing process is very easy and very cheap by means of photoetched procedures.

Fresnel reflectors performance has been widely studied by means of a computer simulator developed by ourselves which is specially adequate and flexible for reflectors with circular symmetry [19-21]. We also have developed a methodology to optimize shaping of Fresnel reflectors [22, 23].

Now, a new simulator has been specifically developed for reconfigurable reflectors composed by squared elements,

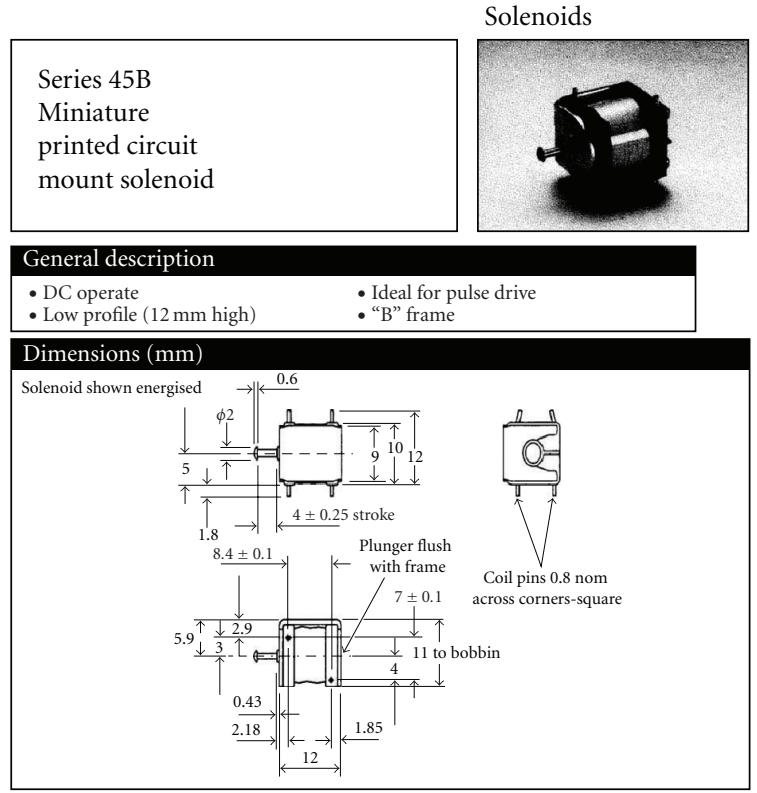

FIgURE 15: Actuator data sheet.

with any number of levels or continuous shifting. In order to get a good efficiency, we have extracted the response of a squared element with uniform illumination. In this way, the response of the reflector is the superposition of the responses of all elements, once situated each one in its location and 

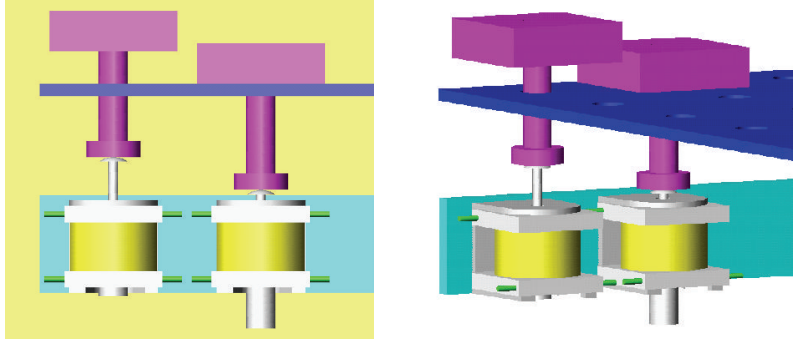

FIGURE 16: Views of two mechanical actuators, working over its respective reflecting cell, at both Fresnel levels: 0 and 1 .

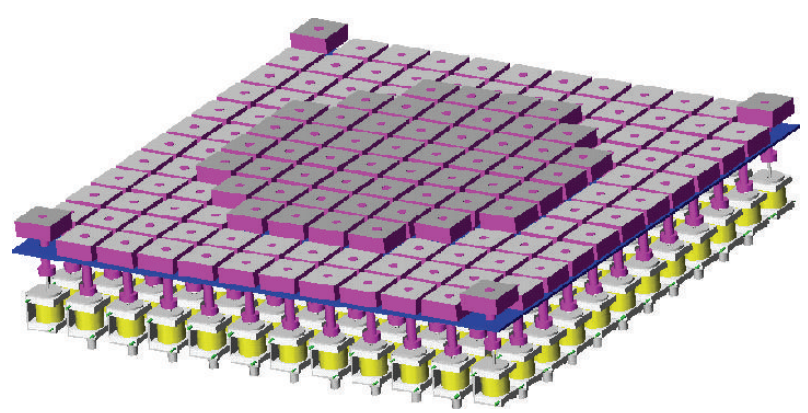

FIGURE 17: View of the active Fresnel reflector with the configuration of Figure 13(b).

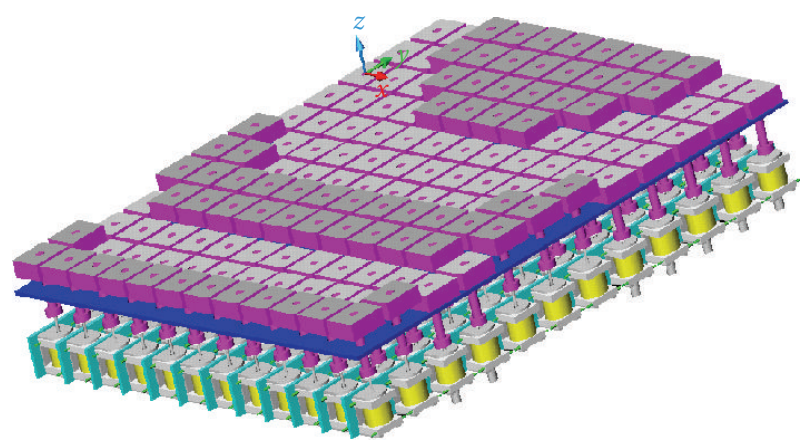

Figure 18: View of the active Fresnel reflector with the configuration of Figure 12.

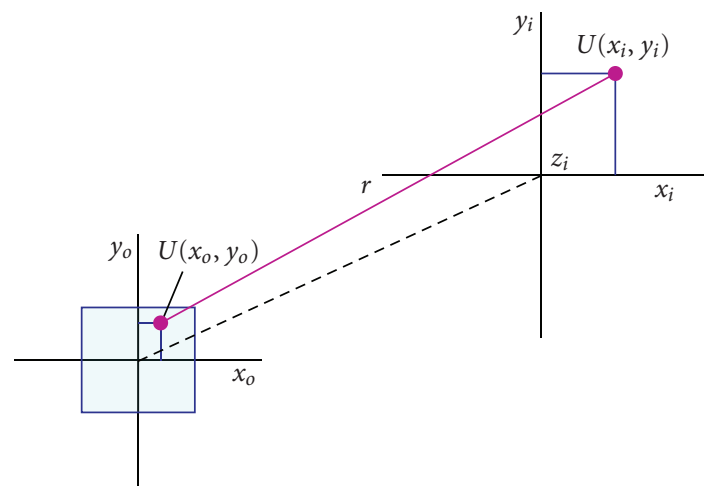

FIGURE 19: Geometry and notation of the radiating cell. level. The assumption that resonance effects and boundary conditions in edges of squared elements are small enough to be neglected is very reasonable because they affect mainly to radiation far from the main lobes. Besides, there are structural media to minimize those effects, as we will try to prove in the future when physical experimentation becomes possible.

On the other hand, the assumption of uniform illumination in the whole area of each conformable element could not be too much closed to the real case; we use a grid to decompose the surface of the reflecting elements in smaller squares (rectangles in general terms) to the end of getting simulation elements small enough to be considered, each one as uniformly illuminated.

Although the normal case is to have squared elements, we are going to consider rectangular ones for giving more generality and flexibility to the simulator.

3.2.1. Rectangular Reflecting Cell. A rectangular surface is used as a reference. The center of this element will be located at $\left(x_{o}, y_{o}, z_{o}\right)=(0,0,0)$ as shown in Figure 19. In this case, the function of luminance of a rectangle with dimensions $a \times h$ can be represented by the following expression (2) $[24,25]:$

$$
U(x, y)=f(x, y) \cdot \Pi\left(\frac{x}{a}\right) \cdot \Pi\left(\frac{y}{h}\right),
$$

being $f(x, y)$ the function of luminance inside the rectangle, and $\Pi(u / b)$ a pulse in axis $u$ of amplitude 1 between $-b / 2$ and $b / 2$. But, if luminance is uniform of amplitude $A$, function (2) is reduced to

$$
U\left(x_{o}, y_{o}\right)=A \cdot \Pi\left(\frac{x_{o}}{a}\right) \cdot \Pi\left(\frac{y_{o}}{h}\right) .
$$

In order to get the far field radiation pattern of the rectangular element, we will use the Fresnel-Kirchhoff diffraction formula [24] as follows:

$$
U\left(x_{i}, y_{i}\right)=\frac{1}{j \lambda} \iint \frac{\exp (j k r)}{r} \cdot U\left(x_{o}, y_{o}\right) \cdot d x_{o} d y_{o} .
$$

According to Figure 19, the distance $r$ of an elementary emitter in $x_{o}, y_{o}$ to an arbitrary far field point in a plane $x_{i}, y_{i}$, parallel to the plane of the radiating element will be calculated as follows:

$$
\begin{aligned}
r^{2} & =z_{i}^{2}+\left(x_{i}-x_{o}\right)^{2}+\left(y_{i}-y_{o}\right)^{2}, \\
z_{i}^{2} & \gg\left(x_{i}-x_{o}\right)^{2}+\left(y_{i}-y_{o}\right)^{2}, \\
r & =z_{i} \cdot \sqrt{1+\frac{\left(x_{i}-x_{o}\right)^{2}+\left(y_{i}-y_{o}\right)^{2}}{z_{i}^{2}}} \\
& \cong z_{i}\left(1+\frac{\left(x_{i}-x_{o}\right)^{2}+\left(y_{i}-y_{o}\right)^{2}}{2 \cdot z_{i}^{2}}\right), \\
r & \cong z_{i} \frac{x_{i}^{2}+y_{i}^{2}}{2 \cdot z_{i}^{2}}-\frac{x_{i} \cdot x_{o}+y_{i} \cdot y_{o}}{z_{i}} .
\end{aligned}
$$




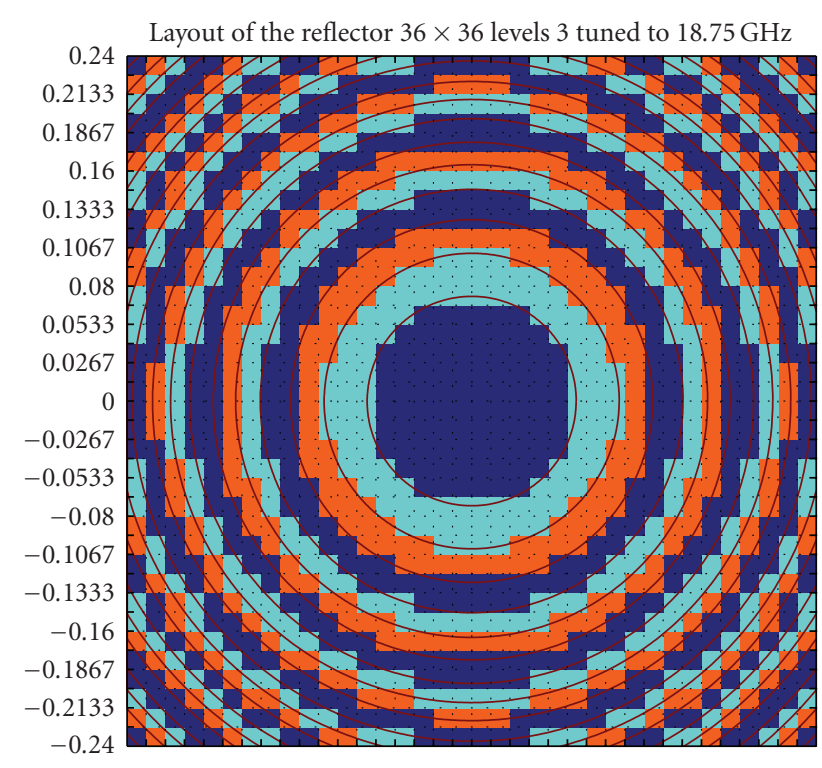

Figure 20: Fresnel reflector of square cells and 3 levels.

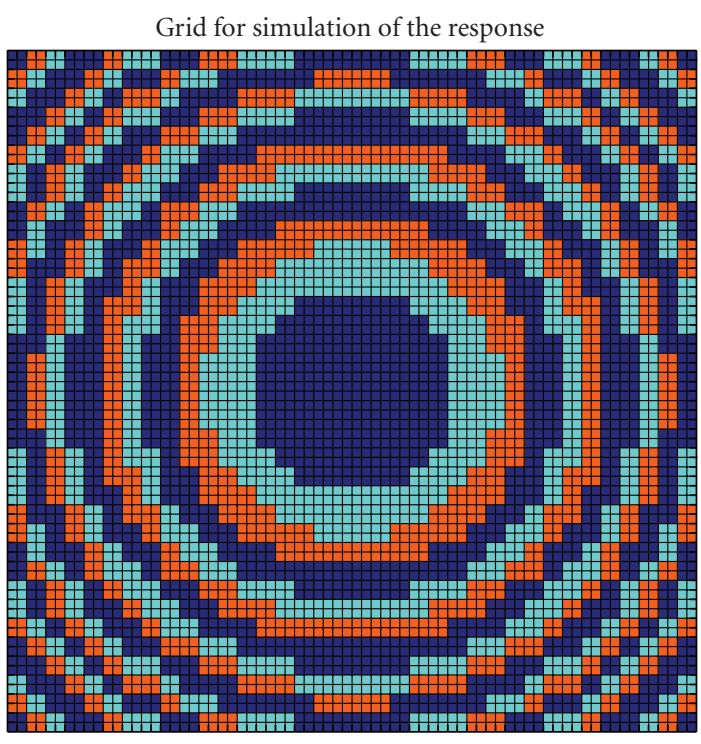

FIGURE 21: Grid for simulation proposal.
Then, introducing this expression of $r$ and the luminance in (4) in the Fresnel-Kirchhoff diffraction formula in (5), we get the far field (Fraunhofer space) due to a rectangular element as follows:

$$
\begin{aligned}
U\left(x_{i}, y_{i}\right)= & \frac{a \cdot h}{j \lambda \cdot z_{i}} \exp \left[j k\left(z_{i}+\frac{\left(x_{i}^{2}+y_{i}^{2}\right)}{2 \cdot z_{i}}\right)\right] \\
& \cdot \operatorname{sinc}\left(a \frac{x_{i}}{\lambda \cdot z_{i}}\right) \cdot \operatorname{sinc}\left(h \frac{y_{i}}{\lambda \cdot z_{i}}\right) .
\end{aligned}
$$

\subsubsection{Fresnel Reflector Conformed by Squared Reflecting Cells.} Once developed a method to get the optimal shape for a flat Fresnel reflector [22], the approaching procedure to obtain a reflector conformed by squared reflecting cells will consist of setting every squared cell at the same level that had its central point in the ideal Fresnel reflector.

As an example, Figure 20 shows the layout of a reflector configured by means of a reconfigurable reflector of $480 \times$ $480 \mathrm{~mm}$ and $36 \times 36$ elements. The shape of the original reflector has been indicated by circles in the same figure. The reflector has been designed at three levels (each level a different color); the focal distance is $500 \mathrm{~mm}$ and has been tuned to $18.75 \mathrm{GHz}$.

It is easy to see in Figure 20 that there is a misalignment between the optimal shape of the Fresnel reflector and that conformed by squares. Of course, increasing the density of elements, they become smaller, and the approximation to the ideal Fresnel reflector, as well as the efficiency of the reflector, is better. However, the reconfigurable system would be more complex and expensive. We will describe below the procedure to get the fair dimensions.

3.2.3. Grid and Feeding Distribution for Simulation. Simulation process has nothing to do with the configuration of the reflector. Any configuration will be simulated by iteratively applying formula (6) for each one of the squared (or rectangular if it were the case) elements once moved to the position $x, y, z$ relative to each point of the de radiation pattern one want to find.

This fact implies that we must know the location, the level $(z)$, and the luminance $A$ of every element, and also that we can suppose that squares are uniformly illuminated. The location and shifting of the elements are well-known parameters. However, the fact of being correct or not to, consider uniform illumination on the surface of the elements, depends on the size of the facets and of the feeding conditions as, for example, radiation pattern of the feeder (or feeders), focal distance, or location and orientation of the feeder.

In order to avoid erroneous considerations about this aspect, the simulator has been designed in such a way that the elements for making simulation can be smaller than the real elements. That is, the real elements can be decomposed into any number of squared or rectangular cells, small enough to be able to consider uniform illumination in each cell.

In other words, the grid used to decompose the surface of the reflector will be smaller than the one defined by the moving facets or squared elements of the reflector. Of course, the simulation cells will be an entire fraction of the moving facets. Figure 21 shows a grid for simulation of the Fresnel reflector whose layout is in Figure 20. As it can be seen, the grid has been made dividing each element into $2 \times 2$ cells.

Once determined the grid for simulation, the next step consists in calculating the intensity of illumination received by all its cells. Knowing the radiation pattern of the feeding system, this calculus is immediate.

As what we want in principle is to know the behavior of a reconfigurable reflector based on squared elements, we are going to consider an ideal feeder which radiates uniformly in any direction but only in the coverage of the aperture of the 


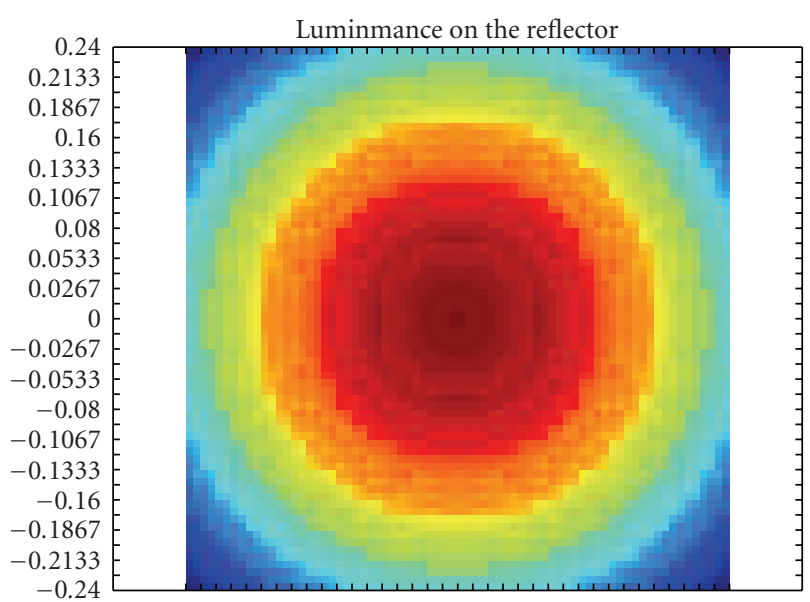

FIgURE 22: Luminance on the grid under ideal feeding conditions.

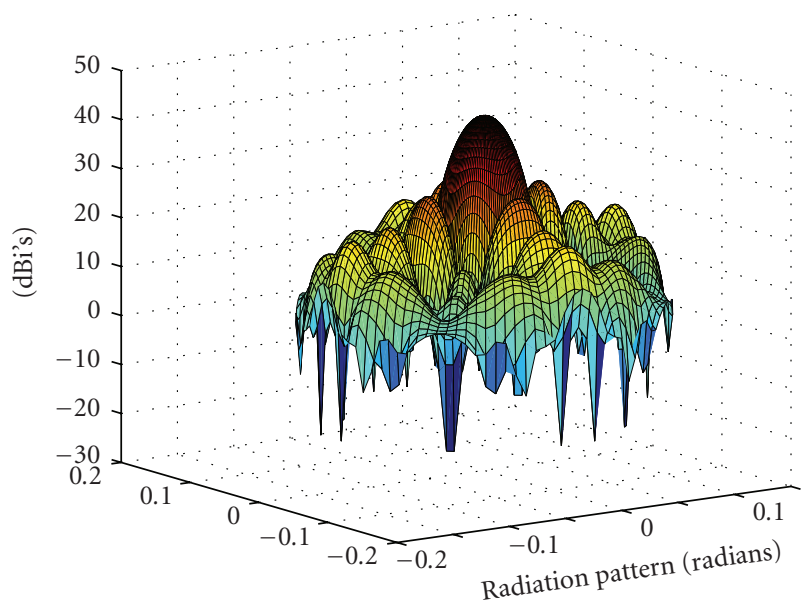

FIgURE 23: Radiation pattern from the 3D simulator.

reflector (squared in this case of study). In Figure 22, it can be seen a color map of the luminance on the grid from the feeder in that conditions.

3.2.4. Response Analysis. As mentioned above, the analysis of the response consists in the superposition of the response of all the cells according to (6). As, in general, the aperture of a reconfigurable reflector can have an arbitrary shape, the radiation pattern will not be symmetrical but will have a different pattern depending on the considered cut. For example, in a squared aperture, as our case of study, the cut of the radiation pattern in a direction parallel to a reflector side will be different from the cut in the direction of a diagonal, or something else. Consequently, it is necessary to specify the angle of the cut to be considered with respect to one of the axis transversal to propagation direction. Another variant we have implemented is the simulator $3 \mathrm{D}$ which is able to represent the full diagram, all around the propagation axis (see Figure 23).

Also, to have an idea about performance of the configuration designed and implemented in the reconfigurable

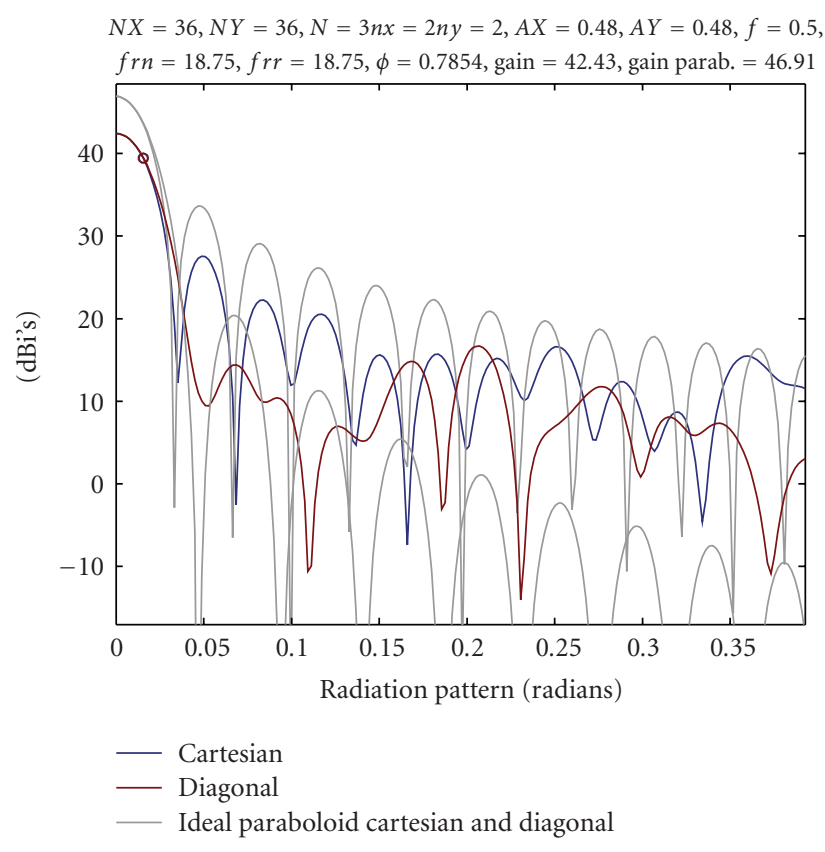

FIGURE 24: Radiation pattern at $0^{\circ}$ and $45^{\circ}$ obtained from developed simulator.

reflector, we will represent the radiation pattern of an absolutely ideal parabolic reflector of the same aperture. The radiation pattern of our case of study as well as of the absolutely ideal paraboloid of the same aperture, in parallel and diagonal directions can be seen in Figure 24.

What we have adopted as absolutely ideal paraboloid is the radiation of its aperture under condition of uniform field in amplitude and phase, that is, the result of applying (6) to the whole aperture. Due to the fact that in our case of study we have not uniform illumination (Figure 22) but uniform radiation of the feeder, a difference of $4.48 \mathrm{~dB}$ from ideal paraboloid (Figure 24) is quite acceptable.

However, if we would conform a paraboloid by means of the reconfigurable reflector (supposed that it was permitted by its structure) in the same conditions of feeding as in the case of the squared Fresnel reflector, the difference only is $1.66 \mathrm{~dB}$ as can be seen in Figure 25. The results of simulation for the conformed Fresnel reflector used as a case of study in $3 \mathrm{D}$ can be seen in Figure 23.

As mentioned above, the selection of the size of the grid is very important for simulation. In fact, if the simulation grid is of the same size as the division in facets of the reconfigurable reflector, simulation can be poor, but if the grid is too much dense, simulation process proves to be very heavy and results can be indistinguishable from those obtained with a less compact grid.

In order to illustrate this simulation effect, we have made simulation of our case of study using grid cells of the same size of the facets $(1 \times 1)$ in comparison with simulation with grid dividing facets in $2 \times 2$ and also with a grid $3 \times 3$. As it can be seen in Figure 26, there is a sensible difference between results from $1 \times 1$ and $2 \times 2$. However, between $2 \times 2$ and $3 \times 3$, difference is negligible. 


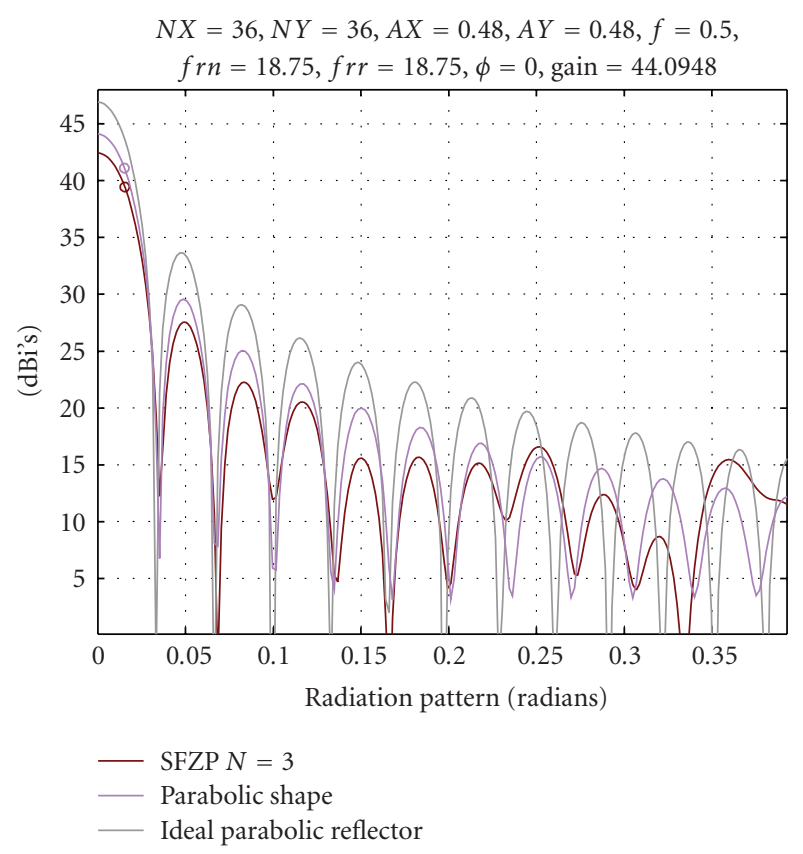

FIGURE 25: Comparison between parabolic shape and Fresnel reflector of squared cells.

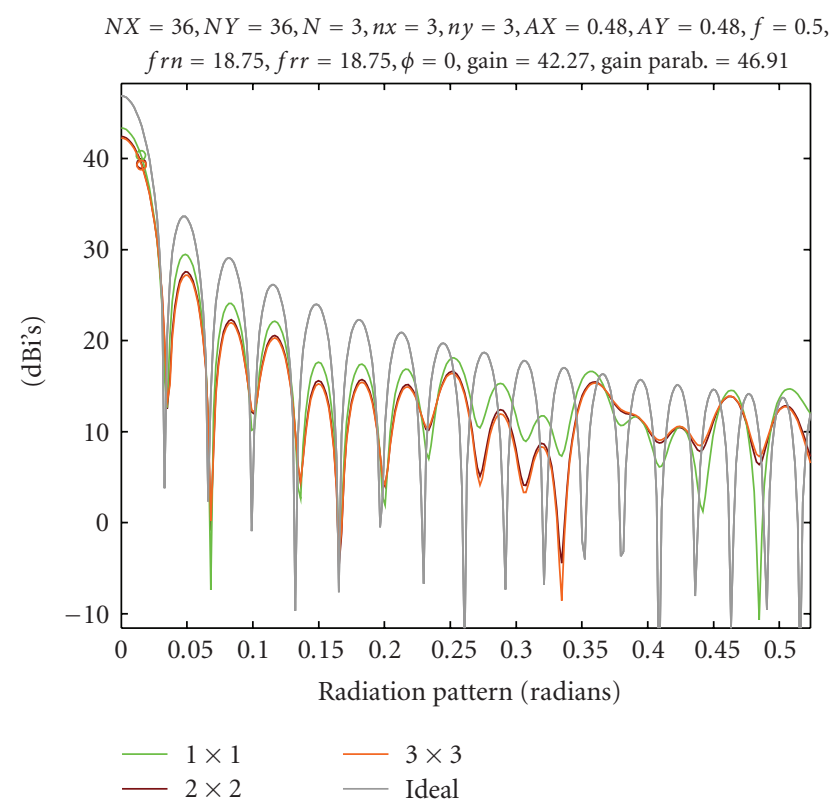

FIgURE 26: Comparison of simulations $1 \times 1,2 \times 2$, and $3 \times 3$.

It is also interesting to evaluate the efficiency of the reconfigurable reflector when conforming Fresnel reflectors, depending on the implemented number of levels, that is, the number of steps that can establish the shifting actuator like that shown in Figure 26 whose number of levels is two. However, it is important to take into account that the efficiency versus number of levels is not independent of the size of the facets of the reconfigurable reflector. As a matter of fact, the greater the number of levels is, the narrower the

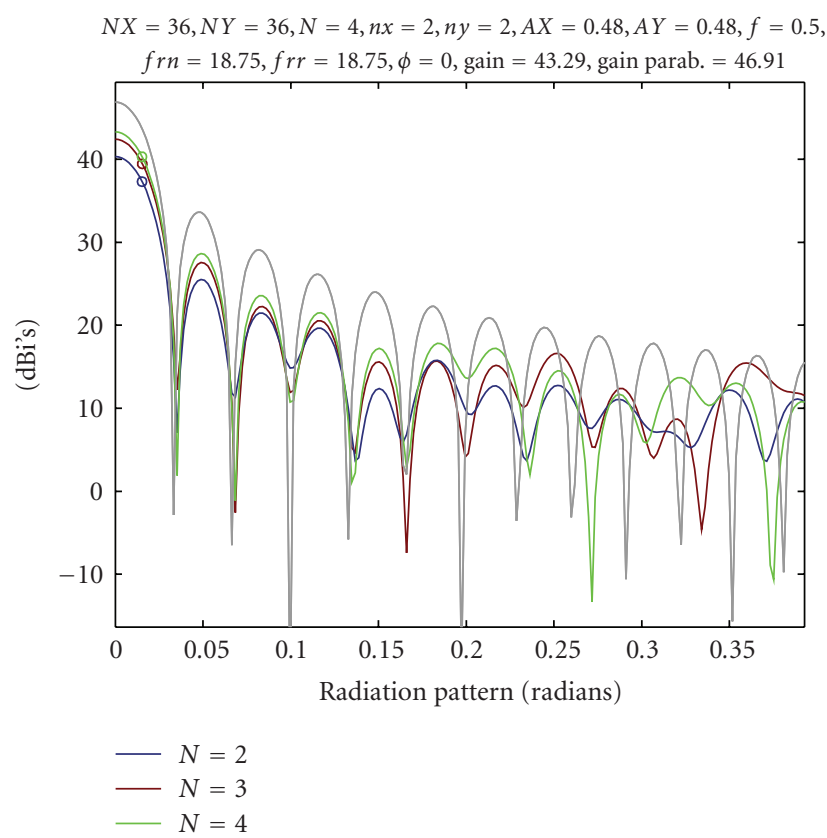

FIGURE 27: Radiation pattern of Fresnel reflectors of squared cells of 2, 3, and 4 levels.

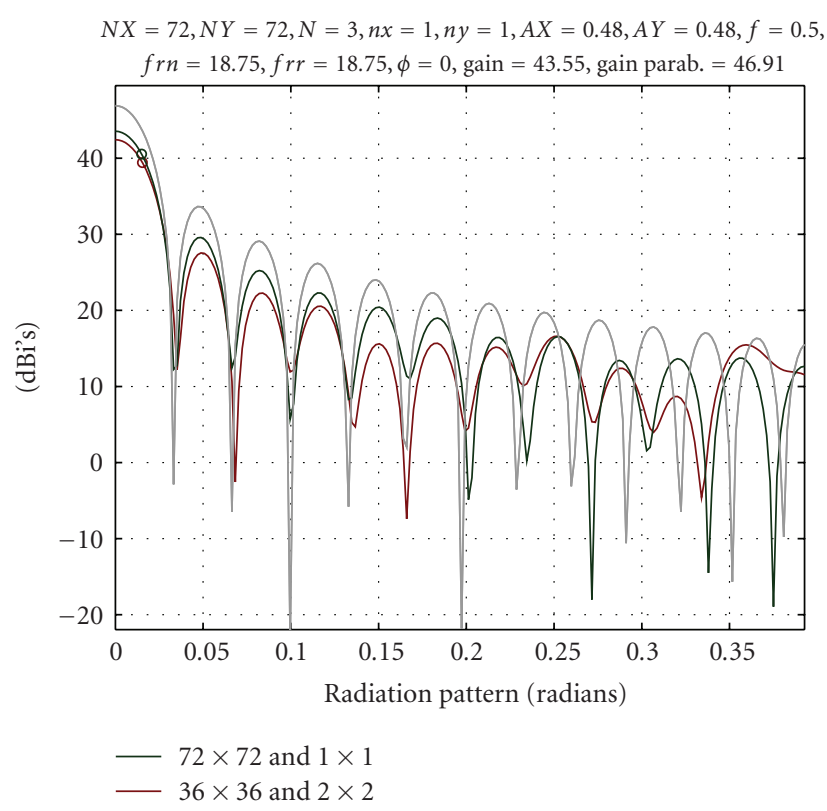

FIGURE 28: Comparison of Fresnel reflectors of $72 \times 72$ and $36 \times 36$ reflecting squared cells, having the same radiating aperture.

size of the Fresnel zones will be. On the contrary, the size of the facets can become wider than many Fresnel zones, which means that the approach to the Fresnel reflector of reference could be very poor. Then, it is necessary to use a size of facets according to the wavelength and to the number of levels to be implemented.

For example, in the initial case of having a reflector of $480 \times 480 \mathrm{~mm}$ with $36 \times 36$ facets, gain varies with the number of implemented levels as follows: $40.3 \mathrm{~dB}$ for two levels $(N=$ 
2), $42.43 \mathrm{~dB}$ for three levels $(N=3)$, and $43.29 \mathrm{~dB}$ for four levels $(N=4)$. In Figure 27, it can be seen the radiation patterns for these three configurations.

However, if we would use a reconfigurable reflector of the same dimensions but with $72 \times 72$ elements instead of $36 \times 36$, performance gets much better since it also betters the approach to the Fresnel reflector of reference. As it can be seen in Figure 28, gain is better with three levels $(N=3)$ in the case of $72 \times 72$ than with higher number of levels $(N=$ 4 ) in the case of $36 \times 36$. Concretely, in the first case gain is $43.55 \mathrm{~dB}$, while in the second it is $43.29 \mathrm{~dB}$. In both cases, the grid of simulation is really of $72 \times 72$ cells, because in the first case each element of simulation is equal to one facet, while in the second one, each facet is divided in $2 \times 2$ simulation cells.

\section{Conclusions}

An active reflectarray with beam steering capabilities has been designed, manufactured, and measured. Based on an IQ configuration, the amplitude and phase of the scattered field for each of its radiating units have been varied. The obtained phase control permits to achieve the required phase shift, not only to convert the spherical incident wave into a plane one but also to steer the main beam. Furthermore, different amplitude distributions with different gains can be easily implemented by using these elements. The measurements of the radiation pattern pointing to different elevation angles have demonstrated the potentiality of the proposed architecture.

On the other hand, a Fresnel reflector configuration can be electronically modified to provide any desired radiation pattern. Beam steering or radiation pattern reconfigurability is some of the possible applications of this new structural concept on active antennas. The design of a square flat reflector with 144 actuators, working at $18.75 \mathrm{GHz}$, shows antenna performance.

Simulation of this kind of reconfigurable reflectors having squared (or rectangular) reflecting cells has also been proved to be reliable and efficient. The procedure has been made by means of the superposition of the radiation of all the elements on a simulation grid. The simulation grid consists in a decomposition of the reflector in a grid of cells submultiple of the elements or facets of the reflector.

Reconfigurable reflectors are very adequate to conform Fresnel plate zones since they are efficient and require only small shifts of the facets. The efficiency of Fresnel reflectors depends on the number of levels implemented and on the size and number of available facets. A reasonable option is to implement three levels with a size of facets adequate for a good sampling of the corresponding reflector profile.

\section{Acknowledgments}

The authors would like to thank the Spanish Ministry of Science and Innovation, by the financial support provided through Projects TIC 2000-0401-P4-09, TIC 2002-04084C03-03, TEC 2005-07985-C03-01/TCM, and TEC 200806684-C03-01/TEC as well as the Collaboration Agreement between the Polytechnical University of Madrid and the Institute of Applied Physics of CSIC to develop new electrical and structural concepts on reflecting antennas. This work is also a result of the collaboration activities in the framework of COST 284 and IC 0603. The authors also thank Andrés de Frutos by his work on drawings and Roque Vassal'lo by the suggestions about the developed prototype of the active Fresnel reflector.

\section{References}

[1] D. M. Pozar, S. D. Targonski, and H. D. Syrigos, "Design of millimeter wave microstrip reflectarrays," IEEE Transactions on Antennas and Propagation, vol. 45, no. 2, pp. 287-296, 1997.

[2] J. Huang, "Capabilities of printed reflectarray antennas," in Proceedings of the IEEE International Symposium on Phased Array Systems and Technology (PAST '96), pp. 131-134, Boston, Mass, USA, October 1996.

[3] M. E. Bialkowski, A. W. Robinson, and H. J. Song, "Design, development, and testing of X-band amplifying reflectarrays," IEEE Transactions on Antennas and Propagation, vol. 50, no. 8, pp. 1065-1076, 2002.

[4] L. Boccia, F. Venneri, G. Amendola, and G. Di Massa, "Experimental investigation of a varactor loaded reflectarray antenna," in Proceedings of the IEEE MTT-S International Microwave Symposium (MWSYM '02), vol. 1, pp. 69-71, Seattle, Wash, USA, June 2002.

[5] S. V. Hum, M. Okoniewski, and R. J. Davies, "Realizing an electronically tunable reflectarray using varactor diode-tuned elements," IEEE Microwave and Wireless Components Letters, vol. 15, no. 6, pp. 422-424, 2005.

[6] P. Ratajczak, P. Brachat, and J.-M. Baracco, "Active reflectarray based on high impedance surface," in Proceedings of the IEEE Antennas and Propagation Society International Symposium (APS '07), pp. 5327-5330, Honolulu, Hawaii, USA, June 2007.

[7] J. Perruisseau-Carrier and A. K. Skrivervik, "Monolithic MEMS-based reflectarray cell digitally reconfigurable over a $360^{\circ}$ phase range," IEEE Antennas and Wireless Propagation Letters, vol. 7, pp. 138-141, 2008.

[8] R. W. Clark, G. H. Huff, and J. T. Bernhard, "An integrated active microstrip reflectarray element with an internal amplifier," IEEE Transactions on Antennas and Propagation, vol. 51, no. 5, pp. 993-999, 2003.

[9] H. D. Hristov, Fresnel Zones in Wireless Links, Zone Plate Lenses and Antennas, Artech House, Boston, Mass, USA, 2000.

[10] A. Pedreira and J. Vassal'lo, Performance Review of Fresnel Zone Flat Reflectors, PIERS, Boston, Mass, USA, 2002.

[11] O. V. Minin and I. V. Minin, Diffractional Optics of Millimeter Waves, IOP Publishing, Bristol, UK, 2004.

[12] D. M. Pozar, "Microstrip antenna aperture-coupled to a microstrip line," Electronics Letters, vol. 21, no. 2, pp. 49-50, 1985.

[13] V. Radisic, Y. Qian, and T. Itoh, "Novel architectures for high-efficiency amplifiers for wireless applications," IEEE Transactions on Microwave Theory and Techniques, vol. 46, no. 11, part 2, pp. 1901-1909, 1998.

[14] D.-C. Chang and M.-C. Huang, "Microstrip reflectarray antenna with offset feed," Electronics Letters, vol. 28, no. 16, pp. 1489-1491, 1992.

[15] D. M. Pozar and T. A. Metzler, "Analysis of a reflectarray antenna using microstrip patches of variable size," Electronics Letters, vol. 29, no. 8, pp. 657-658, 1993. 
[16] J. Huang, "Microstrip reflectarray," in Proceedings of the IEEE Antennas and Propagation Society International Symposium (APS '91), vol. 2, pp. 612-615, London, Canada, June 1991.

[17] J. Vassal'lo, A. Oñoro, F. Ares, et al., "ARCO: a Spanish contribution to the improvement to arrays and reflectarrays with beam control," in Proceedings of the 28th ESA Antenna Workshop on Space Antenna Systems and Technologies, vol. WPP-247, pp. 853-860, Noordwijk, The Netherlands, MayApril 2005.

[18] J. Gutiérrez-Ríos and J. Vassal'lo, "Technological aspects of fresnel zone reflectors," in Advanced on Antennas, Reflectors and Beam Control, chapter 4, pp. 85-113, Research Signpost, Trivandrum, India, 2005.

[19] J. Gutiérrez-Ríos and J. Vassal'lo, "Fresnel zone plate reflectors simulation and radiation diagram analysis," in Proceedings of the 3rd COST 284 Workshop on Innovative Antennas, pp. 1-4, Budapest, Hungary, April 2003.

[20] J. Gutiérrez-Ríos and J. Vassal'lo, "Simulación eficiente de la respuesta de reflectores circulares. aplicación a reflectores de fresnel FZP," in Proceedings of the 18th Simposium Nacional de la Unión Científica Internacional de Radio (URSI '03), Coruña, Spain, September 2003.

[21] J. Gutiérrez-Ríos, J. Vassal'lo, and H. Palacios, "Synthesis and response-analysis of fresnel zone plate reflectors," in Proceedings of the Joint Spanish URSI/COST 284 Workshop, Barcelona, Spain, September 2004.

[22] J. Gutiérrez-Ríos and J. Vassal'lo, "Shape optimization of FZP reflectors," in Proceedings of the 27th ESA Antenna Technology Workshop on Innovative Periodic Antenas: Electromagnetic Bandgap, Left-Handed Materials, Fractal and Frequency Selective Surfaces. WPP-222, Santiago de Compostela, Spain, 2004.

[23] J. Gutiérrez-Ríos and J. Vassal'lo, "Simulated response of Conic Fresnel Zone Plate Reflectors (CFZPS)," in Proceedings of the 1st European Conference on Antennas and Propagation (EuCAP '06), pp. 1-7, Nice, France, November 2006.

[24] K. Iizuka, "Elements of photonics," in Free Space and Special Media, vol. 1, pp. 389-392, John Wiley \& Sons, New York, NY, USA, 2002.

[25] A. Van der Lugt, Opical Signal Processing, John Wiley \& Sons, New York, NY, USA, 1992. 

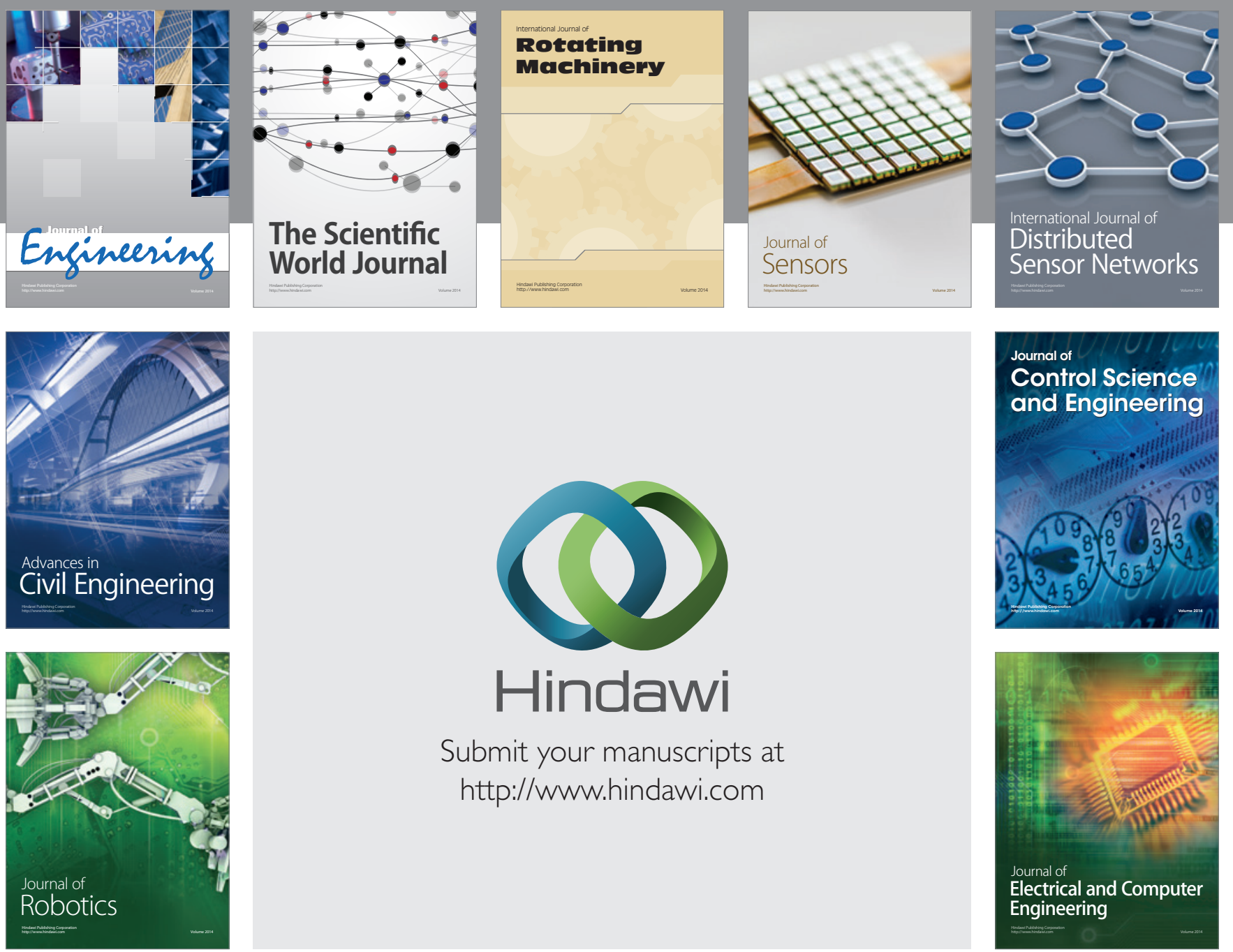

Submit your manuscripts at

http://www.hindawi.com
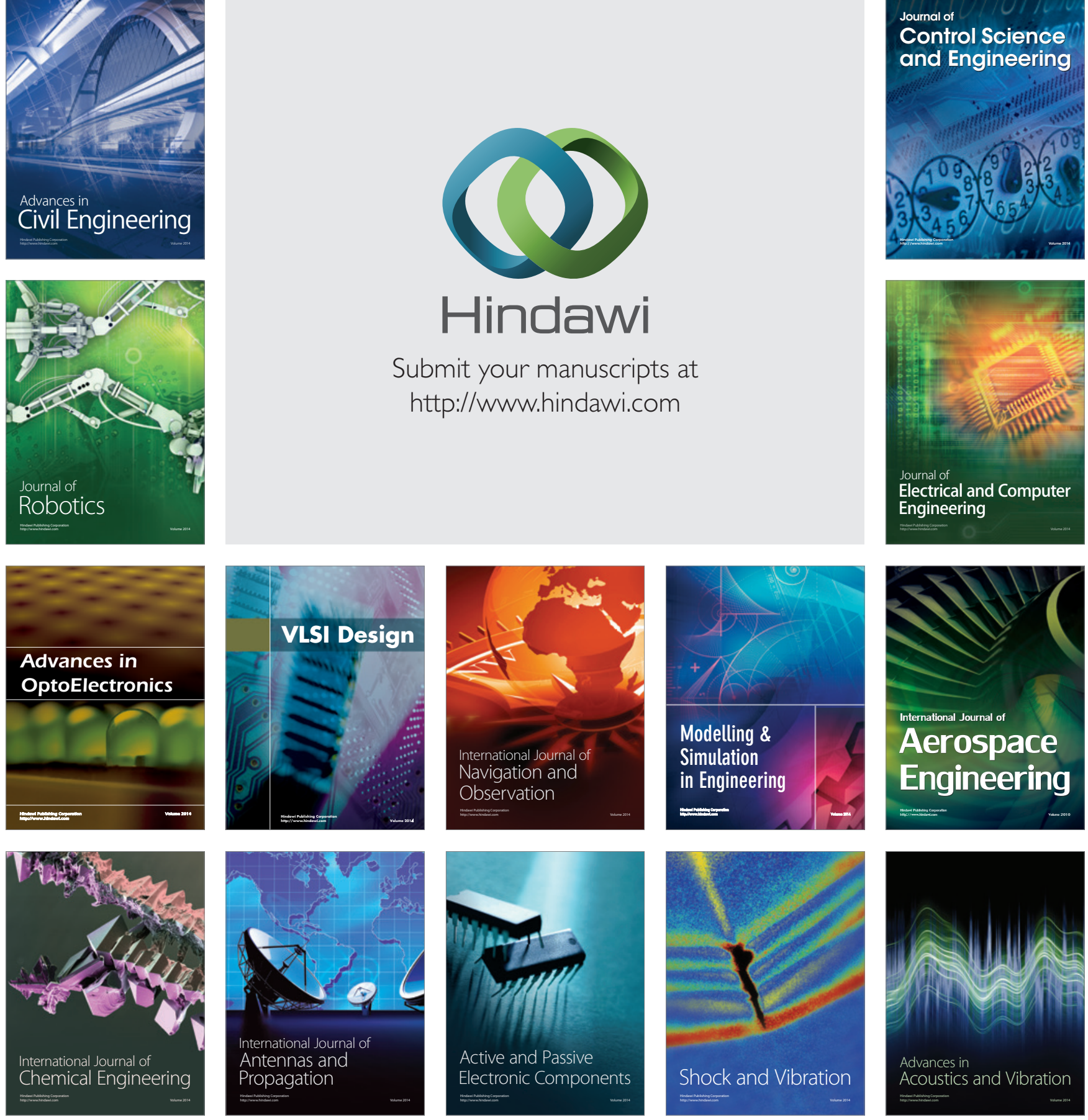